\title{
INVESTIGATION OF THE FLOW-BASED FAST REROUTE MODEL WITH IMPLEMENTATION OF A MULTIMEDIA QUALITY PROTECTION SCHEME
}

\author{
O. Lemeshko', M. Yevdokymenko², O. Yeremenko³, \\ N. Kunicina ${ }^{4}$, A. Ziravecka ${ }^{5}$ \\ 1-3 V.V. Popovskyy Department of Infocommunication Engineering, \\ Kharkiv National University of Radio Electronics \\ 14 Nauky Ave., Kharkiv, 61166, UKRAINE \\ ${ }^{4,5}$ Riga Technical University \\ 12/1 Azenes Str., Riga, LV-1048, LATVIA \\ E-mail: 'oleksandr.lemeshko.ua@ieee.org, \\ ${ }^{2}$ maryna.yevdokymenko@ieee.org, \\ 3oleksandra.yeremenko.ua@ieee.org, \\ ${ }^{4}$ nadezda.kunicina@rtu.Iv, \\ 5anastasija.ziravecka@rtu.Iv
}

In this paper, a tensor flow-based fast reroute model with multimedia quality protection is proposed. In the model, the conditions for implementing a multipath routing strategy and flow conservation are introduced taking into account possible packet loss at the network nodes and preventing overloading communication links both when using the primary and backup routes. At the same time, the novelty of the proposed solution is the formalization of the conditions of protection of the Quality of Experience level in terms of multimedia quality along the primary and backup routes. These conditions have been obtained during the tensor formalization of the network, which made it possible to calculate the quality of service indicators: packet loss probabilities, as well as the average end-to-end delay for audio and video flows transmitted in the multimedia session using the primary and backup routes, respectively. As a criterion for the optimality of the obtained solutions, a condition has been selected related to maximizing the overall performance of the infocommunication network. The results of the research of the proposed model confirm the adequacy of the numerical research results obtained for solving the problem of fast rerouting with link/node protection.

Keywords: Average end-to-end delay, fast rerouting, multimedia quality, packet loss probability, quality of experience, tensor. 
At this stage in the development of infocommunication networks (ICNs), one of the most important requirements put forward by them is to ensure a high level of fault tolerance solutions while maintaining the Quality of Service (QoS). The analysis shows that in practice several approaches are implemented [1]-[10], which are mainly aimed at ensuring either network fault tolerance [1]-[3] or supporting a high level of QoS [4]-[10]. The demand for complex solutions in this area is especially relevant when transmitting multimedia traffic, the volume of which is rapidly growing in modern ICNs.

One of the most effective technological means of ensuring network resiliency is the Fast ReRouting (FRR) protocols [7]-[14]. They should provide a real-time response to possible failures of network equipment based on preliminary calculation and subsequent use of many primary and backup routes with the implementation of local and global protection schemes - a node, link or route. At the same time, it is very important, along with the reservation of its structural elements, to provide protection of the main QoS indicators. Therefore, for example, the works [14]-[16] are devoted to the development of solutions for protecting network bandwidth, and in articles [17]-[22] models and methods are aimed at protecting numerical values of multiple end-to-end QoS indi- cators simultaneously - bandwidth, average delay, packet loss.

However, when transmitting multimedia traffic, for example, voice or online video, it is rather difficult to assess the efficiency of the distribution of network resources and the QoS in the ICN at the end-user level. One way to evaluate QoS for a number of multimedia applications, according to ITU-T [23], [26]-[28], is Mean Opinion Score (MOS), which determines the Quality of Experience (QoE) at the user level. These QoE indicators, taking into account the characteristics of the network, the types of end equipment on the client side, the characteristics of the transmitted multimedia traffic, allow for a more adequate assessment of the quality of the provided multimedia service.

Providing the specified values of QoE indicators using routing tools is a rather complicated scientific and applied problem, requiring a review of not only the relevant protocols but also the mathematical models and methods underlying them, as well as computational algorithms. In this regard, in this paper, the main attention will be paid to the development of an approach that allows, under the conditions of fast rerouting, providing the specified values of QoE indicators along with both the primary and backup routes.

\section{FLOW-BASED FAST REROUTE MODEL WITH PROTECTION OF MILTIMEDIA QUALITY LEVEL IN THE INFOCOMMUNICATION NETWORK}

To protect the level of multimedia quality in conditions of fast rerouting in the ICN, the flow-based model
[24] is used. Here the network structure is presented using a one-dimensional simplicial complex $S=(U, V)$ where 
$U=\left\{u_{i}, i=\overline{1, m}\right\}$ is a set of zero-dimensional simplexes - network nodes (routers) and $V=\left\{v_{z}=(i, j) ; z=\overline{1, n} ; i, j=\overline{1, m} ; i \neq j\right\}$ is a set of one-dimensional simplexes - network links where the link $v_{z}=(i, j)$ models the $z$-th link that connects the routers $u_{i}$ and $u_{j}$. For each link modelled by the edge $v_{z}=(i, j) \in V$, the bandwidth will be denoted both through $\varphi_{z}$ and $\varphi_{i, j}(1 / \mathrm{s})$. Each network router has several interfaces through which it sends packets to its neighbouring nodes. Then $\varphi_{i, j}$ actually determines the bandwidth of the $j$-th interface of the $i$-th node.

Further, we assume that $K$ denotes a set of multimedia sessions on the ICN. Then to solve the QoE-FRR problem, it is necessary to calculate a set of routing variables:

- $x_{i, j}^{k^{s p}}$ and $x_{i, j}^{k^{v i d e o}}$, each characterises the fraction of the rate of the audio and video flows generated during the $k$-th multimedia session and flow in the link $(i, j)$ belonging to the primary route, respectively;

- $\bar{x}_{i, j}^{k^{s p}}$ and $\bar{x}_{i, j}^{k^{\text {video }}}$ are the fractions of the rate of the audio and video flows generated during the $k$-th multimedia session and flow in the link $(i, j)$ belonging to the backup route, respectively.

Then when using a multipath routing strategy, the next limitations are imposed on routing variables:

$\left\{\begin{array}{l}0 \leq x_{i, j}^{k^{s p}} \leq 1, \\ 0 \leq x_{i, j}^{k^{\text {video }}} \leq 1\end{array}\right.$ and $\left\{\begin{array}{l}0 \leq \bar{x}_{i, j}^{k^{s p}} \leq 1, \\ 0 \leq \bar{x}_{i, j}^{k^{\text {video }}} \leq 1 .\end{array}\right.$

To account for possible packet loss caused by overloading the queue buffer on the network nodes, the conditions for conservation of audio and video flows for the primary route takes the following form [24]:

$$
\left\{\begin{array}{l}
\sum_{j:(i, j) \in V} x_{i, j}^{k^{s p}}=1 \text { if } k^{s p} \in K, u_{i}=b_{k} ; \\
\sum_{j:(i, j) \in V} x_{i, j}^{k^{s p}}-\sum_{j:(j, i) \in V} x_{j, i}^{k^{s p}}\left(1-p_{j, i}\right)=0 \text { if } k^{s p} \in K, u_{i} \neq b_{k}, u_{i} \neq d_{k} ; \\
\sum_{j:(j, i) \in V} x_{j, i}^{k^{s p}}\left(1-p_{i, j}\right)=\varepsilon^{k^{s p}} \text { if } k^{s p} \in K, u_{i}=d_{k},
\end{array}\right.
$$

$$
\left\{\begin{array}{l}
\sum_{j:(i, j) \in V} x_{i, j}^{k^{\text {video }}}=1 \text { if } k^{\text {video }} \in K, u_{i}=b_{k} ; \\
\sum_{j:(i, j) \in V} x_{i, j}^{k^{\text {video }}}-\sum_{j:(j, i) \in V} x_{j, i}^{k^{\text {video }}}\left(1-p_{j, i}\right)=0 \text { if } k^{\text {video }} \in K, u_{i} \neq b_{k}, u_{i} \neq d_{k} ; \\
\sum_{j:(j, i) \in V} x_{j, i}^{k^{\text {video }}}\left(1-p_{i, j}\right)=\varepsilon^{k^{\text {video }}} \text { if } k^{\text {video }} \in K, u_{i}=d_{k},
\end{array}\right.
$$

where $b_{k}$ is the source router and $d_{k}$ is the destination router for packets of audio and video flows of the $k$-th multimedia session, respectively; $\varepsilon^{k^{s p}}$ and $\varepsilon^{k^{v t d e o}}$ are the fractions of rate of the audio flow $k^{s p}$ and video flow $k^{v i d e o}$ serviced by the network, i.e., packets of which have been successfully delivered to the destination router; $p_{i, j}$ is the probability of packet loss on the $j$-th interface of the $i$-th router. 
The conditions for conservation of audio and video flows for the backup route have a similar form (2) and (3), where $\bar{p}_{i, j}^{k}$ is the probability of packet loss on the $j$ -th interface of the $i$-th router, which is included in the backup path. The probability of packet loss traditionally depends on the characteristics of the traffic, the discipline of their service, and the state of the interface. The probability of packet loss, if the $j$-th interface of the $i$-th router is modelled by a queuing system with failures of the type, for example, $M / M / 1 / N$, can be calculated as follows:

$p_{i, j}=\frac{\left(1-\rho_{i, j}\right)\left(\rho_{i, j}\right)^{N_{i, j}}}{1-\left(\rho_{i, j}\right)^{N_{i, j}+1}}$ and $\bar{p}_{i, j}=\frac{\left(1-\bar{\rho}_{i, j}\right)\left(\bar{\rho}_{i, j}\right)^{N_{i, j}}}{1-\left(\bar{\rho}_{i, j}\right)^{N_{i, j}+1}}$,

where $\rho_{i, j}=\frac{\lambda_{i, j}}{\varphi_{i, j}}$ and $\bar{\rho}_{i, j}=\frac{\bar{\lambda}_{i, j}}{\varphi_{i, j}}$ are the utilization coefficients of the $j$-th interface on the $i$-th router, which is included in the primary and backup paths, respectively; $N_{i, j}$ denotes the maximum number of packets in the queue of the $j$-th interface on the $i$-th router; $\varphi_{i, j}$ denotes the bandwidth of the $j$-th interface of the $i$-th router, measured in $1 / \mathrm{s} ; \lambda_{i, j}$ and $\lambda_{i, j}$ are the total rate of all flows of various multimedia sessions in the link $(i, j) \in V(1 / \mathrm{s})$ belonging to the primary or backup routes, respectively, calculated using the expression

$$
\left\{\begin{array}{l}
\lambda_{i, j}=\sum_{k \in K}\left(\lambda^{k^{s p}} x_{i, j}^{k^{s p}}+\lambda^{k^{\text {video }}} x_{i, j}^{k^{v i d e o}}\right) \\
\bar{\lambda}_{i, j}=\sum_{k \in K}\left(\bar{\lambda}^{k^{s p}} \bar{x}_{i, j}^{k^{s p}}+\bar{\lambda}^{k^{v i d e o}} \bar{x}_{i, j}^{k^{v i d e o}}\right)
\end{array},\right.
$$

where $\lambda^{k^{s p}}$ and $\lambda^{k^{v i d e o}}$ are the average packet rates of the audio and video flows of the $k$-th multimedia session, respectively, generated along the primary path; $\bar{\lambda}^{k^{s p}}$ and $\bar{\lambda}^{k^{\text {video }}}$ are the average packet rates of the audio and video flows of the $k$-th multimedia session, respectively, generated along the backup path. The rates of successfully transmitted packets of audio $\lambda_{i, j}^{k^{s p}}$ and video $\lambda_{i, j}^{k^{\text {video }}}$ flows of the $k$-th multimedia session through the $j$-th interface of the $i$-th router belonging to the primary route, are calculated as follows:

$\lambda_{i, j}^{k^{s p}}=\lambda^{k_{s p}} x_{i, j}^{k^{s p}}\left(1-p_{i, j}\right)$

$\lambda_{i, j}^{k^{\text {video }}}=\lambda^{k_{\text {video }}} x_{i, j}^{k^{\text {video }}}\left(1-p_{i, j}\right)$

The rate of successfully transmitted packets of audio $\bar{\lambda}_{i, j}^{k^{s p}}$ and video $\bar{\lambda}_{i, j}^{k^{\text {video }}}$ flows of the $k$-th multimedia session through the $j$-th interface of the $i$-th router belonging to the backup route is calculated in similar form as (6) and (7).

To ensure control over the process of overloading links and queues when using the primary and backup routes, taking into account Eq. (5), the following restrictions are introduced into the model structure [24]:

$$
\lambda_{i, j}<\varphi_{i, j} \text { and } \bar{\lambda}_{i, j}<\varphi_{i, j} \text { under }(i, j) \in V \text {. }
$$




\section{CONDITIONS FOR PROTECTION MULTIMEDIA QUALITY IN THE IMPLEMENTATION OF FAST REROUTING}

The main requirement when implementing fast rerouting is to meet the conditions for ensuring a given level of MultiMedia Quality. In accordance with the ITU-T Recommendation G.1070 [19], the requirements for MultiMedia Quality $\left(M M_{q}\right)$ along primary and backup routes, respectively, are generally defined as follows:

$$
\begin{aligned}
& M M_{q} \geq M M_{q}^{<r e q>}, \quad \text { at } 1 \leq M M_{q} \leq 5 . \\
& \overline{M M_{q}} \geq M M_{q}^{<r e q>}, \quad \text { at } 1 \leq \overline{M M_{q}} \leq 5,
\end{aligned}
$$

where

$$
\begin{aligned}
& M M_{q}=m_{1} M M_{S V}+m_{2} M M_{T}+m_{3} M M_{S V} M M_{T}+m_{4}, \\
& \overline{M M_{q}}=m_{1} \overline{M M_{S V}}+m_{2} \overline{M M_{T}}+m_{3} \overline{M M_{S V}} \overline{M M_{T}}+m_{4},
\end{aligned}
$$

where $M M_{q}^{<r e q>}$ is the requirement for the MultiMedia Quality level; $M M_{S V}$ and $\overline{M M_{S V}}$ are the quality of transmission of audiovisual information along primary and backup routes, respectively; $M M_{T}$ and $\overline{M M_{T}}$ denote degradation in quality due to delays and desynchronization of processes for transmitting audio and video flow packets along primary and backup routes, respectively; $m_{i}$ - coefficients depending on the size of the display and the purpose of communication [23], [24].

The main difficulty in ensuring the set values of QoE indicators during fast rerouting was the need for analytical calculation of indicators $M M_{q}$ and $\overline{M M_{q}}$ in order to fulfill conditions (9) and (10) for each multimedia session generated along the primary and the backup routes, respectively. At the same time, in order to calculate these indicators $M M_{q}$ and $\overline{M M_{q}}$ in the framework of a multimedia session when transmitting packets of audio and video flows along the primary and backup routes, it is necessary to calculate the terms presented in accordance with the methodology described in ITU-T G.1070.
Moreover, the terms included in (11) and (12), as well as the transmission quality of audio $\left(S_{q}\right),\left(\bar{S}_{q}\right)$ and video flows $\left(V_{q}\right),\left(\bar{V}_{q}\right)$, are the functions of the average end-to-end delays of packets of audio $\left(T_{S}\right),\left(\bar{T}_{S}\right)$ and video $\left(T_{V}\right),\left(\bar{T}_{V}\right)$ flows, the probabilities of packet losses of audio $\left(P^{S}\right),\left(\bar{P}^{S}\right)$ and video $\left(P^{V}\right),\left(\bar{P}^{V}\right)$ flows along primary and backup routes, respectively, and are determined in accordance with the recommendation [11]. In other words, when transmitting a multimedia flow along the primary route, the transmission quality of audiovisual information $M M_{S V}$ is determined using the following expressions: 


$$
\begin{aligned}
& M M_{S V}=m_{5} S_{q}+m_{6} V_{q}+m_{7} S_{q} V_{q}+m_{8}, \text { at } 1 \leq M M_{S V} \leq 5, \\
& M M_{T}=\max \{A D+M S, 1\}, \text { at } 1 \leq M M_{T} \leq 5, \\
& A D=m_{9}\left(T_{S}+T_{V}\right)+m_{10}, \\
& M S= \begin{cases}\min \left[m_{11}\left(T_{S}-T_{V}\right)+m_{12}, 0\right], & \text { if } \mathrm{T}_{S} \geq T_{V}, \\
\min \left[m_{13}\left(T_{V}-T_{S}\right)+m_{14}, 0\right], & \text { if } \mathrm{T}_{S}<T_{V},\end{cases}
\end{aligned}
$$

where $M S$ is the coefficient that takes into account the desynchronization between the audio and the video; $A D$ is the parameter reflecting the effect of average delays of packets of audio $\left(T_{S}\right)$ and video $\left(T_{V}\right)$ flows. Then, in accordance with recommendation [23], the presented expressions, in this case similar to (13)-(16), can be used to form restrictions of Eq. (9) based on the known required level of quality of multimedia traffic $M M_{q}^{<r e q>}$ transmitted along the primary path. When using the backup route during the transmission of a multimedia flow, expressions similar to (13)-(16) will be used to calculate $\overline{M M}_{S V}$ and $\overline{M M}_{T}$.

The main problem in the calculation $M M_{q}$ and $\overline{M M_{q}}$ is the definition of expressions for finding the values of the end-to-end delays, as well as the packet loss probabilities for audio and video flows (Table 1), transmitted along the primary or backup paths. This is due to the fact that these indicators directly depend on route variables (1), traffic characteristics and network parameters. Therefore, based on the model (1)-(6), the expressions for calculation of $P^{S}, \bar{P}^{S}$ and $P^{V}, \bar{P}^{V}$ will take the form:

$$
\begin{aligned}
& P^{S}=1-\varepsilon^{k^{s p}}, \quad \bar{P}^{S}=1-\bar{\varepsilon} k^{s p}, \\
& P^{V}=1-\varepsilon^{k^{\text {video }}}, \quad \bar{P}^{V}=1-\bar{\varepsilon}^{k^{\text {video }} .}
\end{aligned}
$$

To derive analytical expressions for determining $T_{S}, \bar{T}_{S}$ and $T_{V}, \bar{T}_{V}$ taking into account the results obtained in [20], it is advisable to use the functional of tensor modelling of routing processes in infocommunication networks.

\section{TENSOR FORMALIZATION FAST REROUTE MODEL IN INFOCOMMUNICATION NETWORK}

In accordance with the methodology for tensor modelling of an ICN proposed in [24], [27], the network structure determines the anisotropic space formed by a set of circuits and node pairs. The dimension of this space is determined by the total number of edges (communication links) in the network and is equal to $n$.

Moreover, each independent path (edge, circuit, or node pair) determines the coordinate axis in the space structure. As a rule, an ICN is modelled by a connected onedimensional network, i.e., it contains one connected component. Then the cyclomatic number $\mu$ and the rank $\phi$ of the network determine, respectively, the number of basic circuits and node pairs, for which the following expressions are true: 
$\mu=n-m+1, \phi=m-1, n=\phi+\mu$

In the selected space when transmitting packets of each pair of audio and video flows generated during the $k$-th multimedia session along primary route, the infocommunication network can be represented by a mixed bivalent tensor

$\Omega=T \otimes \Lambda$,

where $\otimes$ is the tensor multiplication operator, the components of the tensor $\Omega$ are the univalent covariant tensor of average packet delays $T$ and the univalent contravariant tensor of flow rates $\Lambda$ in the coordinate paths of the network.

The average packet delay in an arbitrary ICN communication link for both audio and video flows along primary routes is approximated by the expression

$g_{v}^{i i}=\frac{\lambda_{i}\left(1-\left(\rho_{i}^{v}\right)^{N_{i}^{v}+1}\right)\left(1-\rho_{i}^{v}\right) \lambda_{i}^{v}}{\rho_{i}^{v}-\left(\rho_{i}^{v}\right)^{N_{i}^{v}+2}-\left(N_{i}^{v}+1\right)\left(\rho_{i}^{v}\right)^{N_{i}^{v}+1}\left(1-\rho_{i}^{v}\right)}$,
$\tau=\frac{\rho-\rho^{N+2}-(N+1) \rho^{N+1}(1-\rho)}{\lambda\left(1-\rho^{N+1}\right)(1-\rho)}$.

In accordance with the postulate of $\mathrm{G}$. Kron second generalization [25] and the results of [14], [17], [24], Eq. (21) written for each of the network links determines the following vector-matrix equation:

$\Lambda_{v}=G_{v} T_{v}$

where $\Lambda_{v}$ and $T_{v}$ are the projections of the tensors $\Lambda$ and $T$, respectively, in the coordinate systems of the edges represented by the $n$-dimensional vectors of the flow rate and average packet delay in the communication links belonging to the primary routes; $G_{v}=\left\|g_{v}^{i j}\right\|$ is a diagonal $n \times n$ matrix, the elements of which corresponded to the edges (links) of the network and calculated as an example of servicing the audio flow according to Eq. [25]:

where the index $i$ indicates the belonging of a particular interface parameter to the link $v_{i} \in V ; \lambda_{i}$ is the total rate of all flows of various multimedia sessions in the link $v_{i} \in V$ (5); $\lambda_{i}^{v}$ denotes a packet rate of the considered audio flow in the link $v_{i} \in V$. The projections of the tensors of the average packet delays and flow rates in the coordinate system of the circuits and node pairs are connected by the expression similar to (22):

$$
\Lambda_{\pi \eta}=G_{\pi \eta} T_{\pi \eta}
$$

According to the law of coordinate transformation, the tensor $G$ is a twice contravariant metric tensor, the projections of which are transformed as follows when the coordinate system of its consideration is changed:

$G_{\pi \eta}=A^{t} G_{v} A$,

where $G_{\pi \eta}$ is the projection of the tensor
$G$ in the coordinate system of circuits and node pairs; $A$ is the $n \times n$ covariant transformation matrix; $[\cdot]^{t}$ is the transposition operation. As shown in [17], [24], the matrix $G_{\pi \eta}$ can be represented as a block structure, i.e.:

$G_{\pi \eta}=\left\|\begin{array}{lll}G_{\pi \eta}^{\langle 1\rangle} & \mid & G_{\pi \eta}^{\langle 2\rangle} \\ --- & + & --- \\ G_{\pi \eta}^{\langle 3\rangle} & \mid & G_{\pi \eta}^{\langle 4\rangle}\end{array}\right\|, \quad G_{\pi \eta}^{4}=\left\|\begin{array}{lll}G_{\pi \eta}^{\langle 4,1\rangle} & \mid & G_{\pi \eta}^{\langle 4,2\rangle} \\ --- & + & --- \\ G_{\pi \eta}^{\langle 4,3\rangle} & \mid & G_{\pi \eta}^{\langle 4,4\rangle}\end{array}\right\|$, 
where $G_{\pi \eta}^{\langle 1\rangle}$ and $G_{\pi \eta}^{\langle 4\rangle}$ are the square submatrices of the sizes $\mu \times \mu$ and $\phi \times \phi$, respectively; $G_{\pi \eta}^{\langle 2\rangle}$ is the submatrix of the size $\mu \times \phi ; G_{\pi \eta}^{\langle 3\rangle}$ is the submatrix of the size $\phi \times \mu ; G_{\pi \eta}^{\langle 4,1\rangle}$ is the first element of the matrix $G_{\pi \eta}^{\langle 4\rangle} ; G_{\pi \eta}^{\langle 4,2\rangle}$ is the second element of the matrix $G_{\pi \eta}^{\langle 4\rangle}$ of the size $1 \times(\phi-1)$; $G_{\pi \eta}^{\langle 4,3\rangle}$ is the third element of the matrix $G_{\pi \eta}^{\langle 4\rangle}$ of the size $(\phi-1) \times 1$; and $G_{\pi \eta}^{\langle 4,4\rangle}$ is the fourth element of the matrix $G_{\pi \eta}^{\langle 4\rangle}$ of the size $(\phi-1) \times(\phi-1)$.

In the framework of the tensor description of the infocommunication network in the context of the multipath routing strategy [14], [17], [24], the average end-to-end delay of the audio flow packets along the primary route can be calculated as follows:

$$
T_{S}=\frac{\lambda^{k^{s p}} \varepsilon^{k^{s p}}-G_{\pi \eta}^{\langle 4,2\rangle}\left[G_{\pi \eta}^{\langle 4,4\rangle}\right]^{-1} \Lambda_{\eta-1}^{k^{s p}}}{\left(G_{\pi \eta}^{\langle 4,1\rangle}-G_{\pi \eta}^{\langle 4,2\rangle}\left[G_{\pi \eta}^{\langle 4,4\rangle}\right]^{-1} G_{\pi \eta}^{\langle 4,3\rangle}\right)},
$$

where $\Lambda_{\zeta-1}^{k^{s p}}$ is the rate vector of lost packets on the interfaces of routers, the coordinates of which are determined by the expression:

$\lambda_{\eta}^{i}=\sum_{j=1}^{m} \lambda^{k^{s p}} x_{i, j}^{k^{s p}} p_{i, j}$
To determine the average end-to-end delay of the video flow $T_{V}$ packets for the primary route, one can use expressions similar to (26) and (27) with some correction of the metric (23), in which $\lambda_{i}^{v}$ will already numerically characterize the packet intensity of the considered video flow in the link $v_{i} \in V$. Thus, the average end-to-end delay of video flow $T_{V}$ packets along primary route is calculated as follows:

$T_{V}=\frac{\lambda^{k^{\text {video }}} \varepsilon^{k^{\text {video }}}-G_{\pi \eta}^{\langle 4,2\rangle}\left[G_{\pi \eta}^{\langle 4,4\rangle}\right]^{-1} \Lambda_{\eta-1}^{k^{\text {video }}}}{G_{\pi \eta}^{\langle 4,1\rangle}-G_{\pi \eta}^{\langle 4,2\rangle}\left[G_{\pi \eta}^{\langle 4,4\rangle}\right]^{-1} G_{\pi \eta}^{\langle 4,3\rangle}}$,

where $\Lambda_{c-1}^{k^{\text {video }}}$ is the intensity vector of the lost packets of the video flow on the interfaces of the routers along the primary route, the coordinates of which are determined similarly to Eq. (27).

The technique for obtaining the required conditions for ensuring the quality of service by the average end-to-end delay of audio $\bar{T}_{S}$ and video $\bar{T}_{V}$ flow packets along the backup routes is similar to that described above (19)-(28).

As a criterion for the optimality of the obtained solutions in term QoE-FRR, we selected a condition, which allowed maximizing the overall performance of the infocommunication network [24]:

$\max _{x, \varepsilon} \sum_{k \in K}\left(\lambda^{k^{s p}} \varepsilon^{k^{s p}}+\bar{\lambda}^{k^{s p}} \bar{\varepsilon}^{k^{s p}}+\lambda^{k^{\text {video }}} \varepsilon^{k^{\text {video }}}+\bar{\lambda} k^{\text {video }} \bar{\varepsilon}^{\text {video }}\right)$

in the presence of restrictions (1), (2), (5), (9), considering their detail in (10)-(29).

\section{INVESTIGATION AND DISCUSSION OF FRR MODEL WITH MULTIMEDIA QUALITY PROTECTION}

To assess the adequacy of the proposed model (1)-(30) and the demonstrativeness of the obtained calculation results, we will solve this problem for a fragment of the 
infocommunication network as shown in Figs. 1 and 2. Let us assume that the network under investigation consists of sixteen routers and twenty-four communication links, indicating their capacity $(1 / \mathrm{s})$ in the gaps of the links.

Let the packet audio $k^{s p}$ and video $k^{\text {video }}$ flow be transmitted between the first and sixteenth routers with the following QoE requirements: $\lambda^{k^{s p(\text { req })}}=1001 / \mathrm{s}, \lambda^{k^{\text {video }(\text { req })}}=300$ $1 / \mathrm{s}, \quad M M q^{\langle r e q\rangle} \geq 3.5, \overline{M M q}^{\langle r e q\rangle} \geq 3.5$ for primary (Fig. 1) and backup (Fig. 2) multipath in ICN accordingly, when some users are dissatisfied [10].

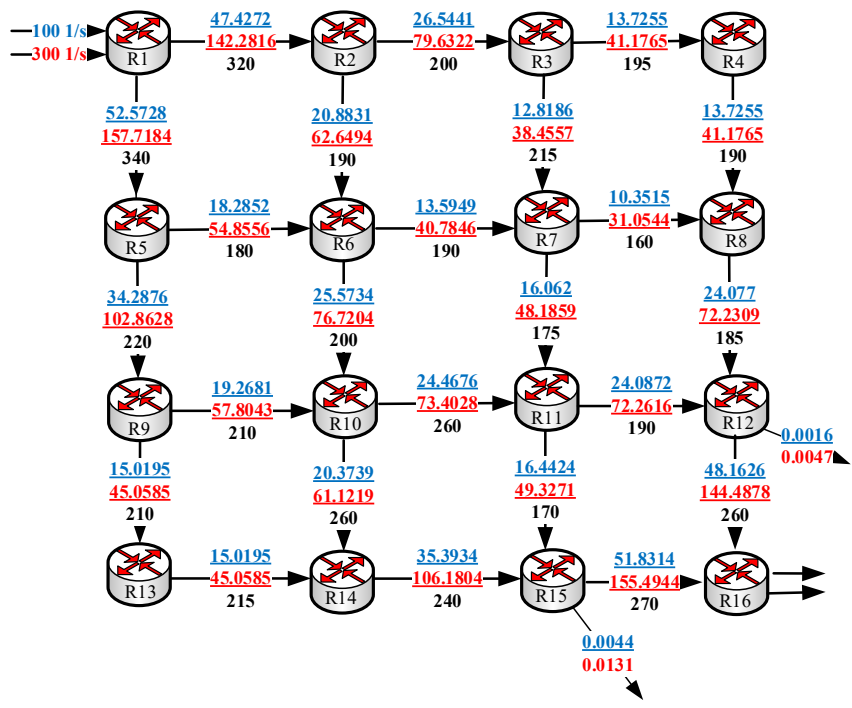

Fig. 1. The routing order of a flow of audio and video packets that is transmitted along the primary multipath.

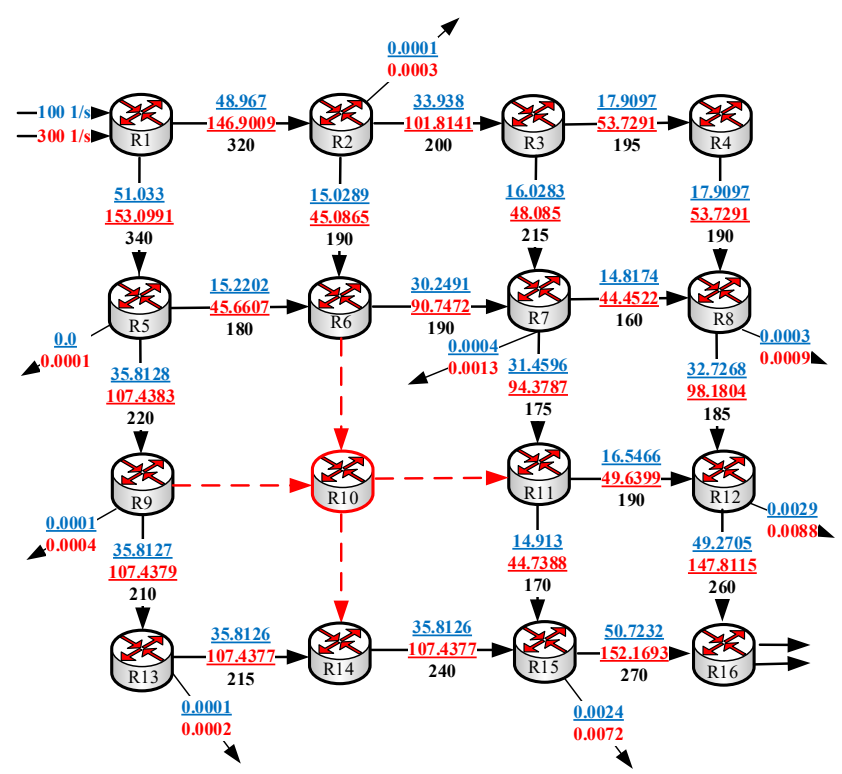

Fig. 2. The routing order of a flow of audio and video packets that is transmitted along the backup multipath. 
In accordance with the presented initial data and MMq requirements for primary multipath, the solution to the fast rerouting problem has been obtained using the proposed FRR model (1)-(29), the results of which are presented in Table 1.

Table 1. Results of Solving the Problem of Providing MMq along the Primary Multipath

\begin{tabular}{|c|c|c|c|c|c|}
\hline \multirow{3}{*}{ Link } & \multirow{3}{*}{$\varphi_{i, j}, 1 / \mathrm{s}$} & \multicolumn{4}{|c|}{ 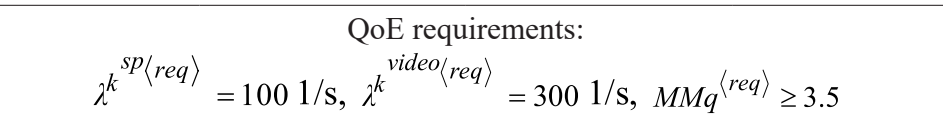 } \\
\hline & & \multicolumn{2}{|c|}{$\begin{array}{l}\text { Calculation results } \\
\text { for the audio flow }\end{array}$} & \multicolumn{2}{|c|}{$\begin{array}{l}\text { Calculation results } \\
\text { for the video flow }\end{array}$} \\
\hline & & $\lambda_{i, j}^{k^{s p}}, 1 / \mathrm{s}$ & $r_{i, j}^{k^{s p}}, 1 / \mathrm{s}$ & $\lambda_{i, j}^{k^{v i d e o}}, 1 / \mathrm{s}$ & $r_{i, j}^{k^{\text {video }}, 1 / \mathrm{s}}$ \\
\hline$(1,2)$ & 320 & 47.4272 & 0 & 142.2816 & 0 \\
\hline$(1,5)$ & 340 & 52.5728 & 0 & 157.7184 & 0 \\
\hline$(2,3)$ & 200 & 26.5441 & 0 & 79.6322 & 0 \\
\hline$(2,6)$ & 190 & 20.8831 & 0 & 62.6494 & 0 \\
\hline$(3,4)$ & 195 & 13.7255 & 0 & 41.1765 & 0 \\
\hline$(3,7)$ & 215 & 12.8186 & 0 & 38.4557 & 0 \\
\hline$(4,8)$ & 190 & 13.7255 & 0 & 41.1765 & 0 \\
\hline$(5,6)$ & 180 & 18.2852 & 0 & 54.8556 & 0 \\
\hline$(5,9)$ & 220 & 34.2876 & 0 & 102.8628 & 0 \\
\hline$(6,7)$ & 190 & 13.5949 & 0 & 4037846 & 0 \\
\hline$(6,10)$ & 200 & 25.5734 & 0 & 76.7204 & 0 \\
\hline$(7,8)$ & 160 & 10.3515 & 0 & 31.0544 & 0 \\
\hline$(7,11)$ & 175 & 16.062 & 0 & 48.1859 & 0 \\
\hline$(8,12)$ & 185 & 24.077 & 0 & 72.2309 & 0 \\
\hline$(9,10)$ & 210 & 19.2681 & 0 & 51.8043 & 0 \\
\hline$(9,13)$ & 210 & 15.0195 & 0 & 45.0585 & 0 \\
\hline$(10,11)$ & 260 & 24.4676 & 0 & 73.4028 & 0 \\
\hline$(10,14)$ & 260 & 20.3739 & 0 & 61.1219 & 0 \\
\hline$(11,12)$ & 190 & 24.0872 & 0 & 72.2616 & 0 \\
\hline$(11,15)$ & 170 & 16.4424 & 0 & 49.3271 & 0 \\
\hline$(12,16)$ & 260 & 48.1626 & 0.0016 & 144.4878 & 0.0047 \\
\hline$(13,14)$ & 215 & 15.0195 & 0 & 45.0585 & 0 \\
\hline$(14,15)$ & 240 & 35.3934 & 0 & 106.1804 & 0 \\
\hline$(15,16)$ & 270 & 51.8314 & 0.0044 & 155.4944 & 0.0131 \\
\hline
\end{tabular}

The results of calculation of MMq level initial requirements are presented in Table 2 . along the backup multipath according to the 
Table 2. Results of Solving the Problem of Providing MMq along the Backup Multipath

\begin{tabular}{|c|c|c|c|c|c|}
\hline \multirow{3}{*}{ Link } & \multirow{3}{*}{$\begin{array}{c}\varphi_{i, j}, \\
1 / \mathrm{s}\end{array}$} & \multicolumn{4}{|c|}{$\begin{array}{l}\text { QoE requirements: } \\
/ \mathrm{s}, \bar{\lambda}^{k^{\text {video }\langle\text { req }\rangle}}=3001 / \mathrm{s}, \overline{M M q}^{\langle\text {req }\rangle} \geq 3.5\end{array}$} \\
\hline & & \multicolumn{2}{|c|}{ Calculation results for the audio flow } & \multicolumn{2}{|c|}{ Calculation results for the video flow } \\
\hline & & $\bar{\lambda}_{i, j}^{k^{s p}}, 1 / \mathrm{s}$ & $\bar{r}_{i, j}^{k p}, 1 / \mathrm{s}$ & $\bar{\lambda}_{i, j}^{k^{\text {video }}, 1 / \mathrm{s}}$ & $\bar{r}_{i, j} k^{\text {video }}, 1 / \mathrm{s}$ \\
\hline$(1,2)$ & 320 & 48.967 & 0 & 146.9009 & 0 \\
\hline$(1,5)$ & 340 & 51.033 & 0 & 153.0991 & 0 \\
\hline$(2,3)$ & 200 & 33.938 & 0.0001 & 101.8141 & 0.0003 \\
\hline$(2,6)$ & 190 & 15.0289 & 0 & 45.0865 & 0 \\
\hline$(3,4)$ & 195 & 17.9097 & 0 & 53.7291 & 0 \\
\hline$(3,7)$ & 215 & 16.0283 & 0 & 48.085 & 0 \\
\hline$(4,8)$ & 190 & 17.9097 & 0 & 53.7291 & 0 \\
\hline$(5,6)$ & 180 & 15.2202 & 0 & 45.6607 & 0 \\
\hline$(5,9)$ & 220 & 35.8128 & 0 & 107.4383 & 0.0001 \\
\hline$(6,7)$ & 190 & 30.2491 & 0 & 90.7472 & 0 \\
\hline$(6,10)$ & 0 & 0 & 0 & 0 & 0 \\
\hline$(7,8)$ & 160 & 14.8174 & 0 & 44.4522 & 0 \\
\hline$(7,11)$ & 175 & 31.4596 & 0.0004 & 94.3787 & 0.0013 \\
\hline$(8,12)$ & 185 & 32.7268 & 0.0003 & 98.1804 & 0.0009 \\
\hline$(9,10)$ & 0 & 0 & 0 & 0 & 0 \\
\hline$(9,13)$ & 210 & 35.8127 & 0.0001 & 107.4379 & 0.0004 \\
\hline$(10,11)$ & 0 & 0 & 0 & 0 & 0 \\
\hline$(10,14)$ & 0 & 0 & 0 & 0 & 0 \\
\hline$(11,12)$ & 190 & 16.5466 & 0 & 49.6399 & 0 \\
\hline$(11,15)$ & 170 & 14.913 & 0 & 44.7388 & 0 \\
\hline$(12,16)$ & 260 & 49.2705 & 0.0029 & 147.8115 & 0.0088 \\
\hline$(13,14)$ & 215 & 35.8126 & 0.0001 & 107.7377 & 0.0002 \\
\hline$(14,15)$ & 240 & 35.8126 & 0 & 107.4377 & 0 \\
\hline$(15,16)$ & 270 & 50.7232 & 0.0024 & 152.1693 & 0.0072 \\
\hline
\end{tabular}

As a result of the calculations (see Table 1, Table 2), which quantitatively meet the requirements for ensuring the level of multimedia quality in the network along the pri- mary multipath (9) at $M M q^{\langle r e q\rangle} \geq 3.5$, and along the backup multipath, the following values obtained are presented in Table 3. 
Table 3. Results of Solving the Problem of Providing FRR with the Level of MMq in ICN

\begin{tabular}{|l|c|c|}
\hline & $\begin{array}{c}\text { Calculation results for the } \\
\text { MMq value along the primary } \\
\text { multipath }\end{array}$ & $\begin{array}{c}\text { Calculation results for } \\
\text { the MMq value along the } \\
\text { backup multipath }\end{array}$ \\
\hline Multimedia quality & $M M q=3.5083$ & $\overline{M M q}=3.5004$ \\
\hline $\begin{array}{l}\text { The transmission quality of audiovisual infor- } \\
\text { mation for audio and video flow packets }\end{array}$ & $M M_{S V}=3.3539$ & $\overline{M M}_{S V}=3.3530$ \\
\hline $\begin{array}{l}\text { The degradation in quality due to delays and } \\
\text { desynchronization of processes for transmit- } \\
\text { ting audio and video flow packets }\end{array}$ & $M_{T}=3.8768$ & $\overline{M M}_{T}=3.8668$ \\
\hline $\begin{array}{l}\text { The average delays of packets of audio and } \\
\text { video flows, 1/s }\end{array}$ & $T_{S}=T_{V}=0.059$ & $\bar{T}_{S}=\bar{T}_{V}=0.0745$ \\
\hline $\begin{array}{l}\text { The probabilities of the total losses of packets } \\
\text { of audio and video flows }\end{array}$ & $P^{S}=P^{V}=0.001$ & $\bar{P}^{S}=\bar{P}^{V}=0.009$ \\
\hline Evaluation of MMq level [19] & Some users dissatisfied & Some users dissatisfied \\
\hline
\end{tabular}

Among the results of the calculations shown in Table 3, it was possible to provide a given level of multimedia quality along the primary (Table 1) and backup (Table 2) multipath. The level of multimedia quality through the backup path $(\overline{M M q}=3.5004)$ was slightly lower than the primary path $(M M q=3.5083)$. This was determined by the fact that when calculating the backup path in the network structure was without router $R 10$ and incident links to it, which affected the bandwidth of the network in general. A characteristic feature of the routing solutions obtained is that the average packet delays along routes that formed a single primary (Fig. 1) or separately backup (Fig. 2) multipath were the same for both audio and video flows. This effect was positive about the value of Eq. (16) associated with the decryption of the audio and video flow packet arrivals that were transmitted within a single multimedia session.

\section{CONCLUSIONS}

In this paper, a tensor flow-based model of fast rerouting with protection of the multimedia quality level has been proposed. This fast rerouting model belongs to the class of flow-based models and includes the conditions for implementing a multipath routing strategy (1), the conditions for conservation of audio and video flows taking into account possible losses at the network nodes (2) and preventing overloading of links (6) both when using the primary and backup routes. At the same time, the novelty of the proposed solution is ensuring the level of multimedia quality not only along the primary route, but also along the backup one. This has been achieved by analytically calculating the QoS indicators: packet loss probabilities for audio and video flows (17), (18), as well as the average end-to-end delays (26), (28) for the same flows transmitted within the multimedia session when using primary and backup routes, respectively. Expressions (26), (28) characterizing the average end-to-end delay of audio and video flows of packets, respectively, along the primary route, as well as similar expressions for calculating this QoS indicator when using the backup route, have been obtained using the tensor research methodology. Thus, the possibility of an analytical 
and interconnected calculation of end-toend QoS indicators (25) allows controlling the impact of time desynchronization in the delivery processes of packets of audio and video flows (14)-(16) on Multimedia Quality using the primary (9) and backup (10) routes, which is very important in the practical implementation of the proposed routing model.

The research of the proposed model with multimedia quality protection has been carried out on the example of the network presented in Figs. 1 and 2. The results of the research have confirmed the adequacy of the calculated results obtained to solve the problem of fast rerouting with link/node protection. Due to the introduction of conditions (9) into the structure of the model and control over the values of the average end-to-end packet delay (26) and (28), as well as the probability of packet loss (17), (18), it was possible to provide a set level of multimedia quality along both the primary (Table 1) and the backup routes (Table 2).

\section{REFERENCES}

1. Savva, S. (2017). Fault-tolerant routing methodology for Networks-on-Chip. In 27th International Symposium on Power and Timing Modeling, Optimization and Simulation (PATMOS), (pp. 1-3), 25-27 September 2017, Thessaloniki, Greece. DOI:10.1109/PATMOS.2017.8106993

2. Ren, Y., Liu, L., Yin, S., Han, J., Wu, Q., \& Wei, S. (2013). A Fault Tolerant NoC Architecture Using Quad-Spare Mesh Topology and Dynamic Reconfiguration. Journal of Systems Architecture. Elsevier, 59 (7), 482-491. DOI:10.1016/j. sysarc. 2013.03.010

3. Papán, J., Segeč, P., Moravčík, M., Hrabovský, J., Mikuš, L., \& Uramova, J. (2017). Existing mechanisms of IP fast reroute. In 15th International Conference on Emerging eLearning Technologies and Applications (ICETA), (pp. 1-7), 26-27 October 2017, Stary Smokovec. DOI:10.1109/ICETA.2017.8102516

4. Seufert, M., \& Tran-Gia, P. (2018). Quality of experience and access network traffic management of HTTP adaptive video streaming. In IEEE/IFIP Network Operations and Management Symposium, (pp. 1-8), 23-27 April 2018, Taipei. DOI:10.1109/NOMS.2018.8406136
5. Liotou, E., Tsolkas, D., \& Passas, N. (2016). A roadmap on QoE metrics and models. In 23rd International Conference on Telecommunications (ICT), (pp. 1-5), 16-18 May 2016, Thessaloniki, Greece. DOI:10.1109/ICT.2016.7500363

6. Litvinenko, A., \& Aboltins, A. (2016). Selection and performance analysis of chaotic spreading sequences for DS-CDMA systems. In Advances in Wireless and Optical Communications (RTUWO), (pp. 38-45), 3-4 November 2016, Riga, Latvia. DOI: 10.1109/RTUWO.2016.7821852

7. Kondratjevs, K., Kunicina, N., Patlins, A., Zabasta, A., \& Galkina, A. (2016). Vehicle weight detection sensor development for data collecting in sustainable city transport system. In 57th International Scientific Conference on Power and Electrical Engineering of Riga Technical University (RTUCON), (pp. 1-5), 13-14 October 2016, Riga, Latvia. DOI: 10.1109/ RTUCON.2016.7763136

8. Dorogovs, P., \& Romanovs, A. (2015). Overview of government e-service security challenges. In IEEE 3rd Workshop on Advances in Information, Electronic and Electrical Engineering (AIEEE), (pp. 1-5), 13-14 Novenber 2015, Riga, Latvia. DOI: 10.1109/AIEEE.2015.7367316 
9. Gasparjans, A., Terebkov, A., \& Žiravecka, A. (2017) Application of Resonance Method for Examining of Piston Ring Technical Condition. In 16th International Scientific Conference "Engineering for Rural Development”, (pp.884-891), 24-26 May, 2017, Latvia, Jelgava. DOI:10.22616/ ERDev2017.16.N180

10. Hands, D.S. (2001). A basic multimedia quality model. IEEE Transactions on Multimedia, 6 (6), 806-816. DOI: https:// doi.org/10.1109/TMM.2004.837233

11. Lemeshko, O., Yeremenko, O., Yevdokymenko, M., Nevzorova, O., Snihurov, A., \& Kovalenko, T. (2019). Fast reroute model with VoIP quality of experience protection. In IEEE 3rd International Conference on Advanced Information and Communications Technologies (AICT), (pp. 16-21), 2-3 July 2019, Lviv, Ukraine. DOI: 10.1109/ AIACT.2019.8847918.

12. Churyumov, G., Tkachov, V.M., Tokariev, V., \& Diachenko, V. (2018). Method for Ensuring Survivability of Flying Adhoc Network Based on Structural and Functional Reconfiguration. In XVIII International Scientific and Practical Conference "Information Technologies and Security” (ITS), (pp. 64-76), 27 November 2018, Ukraine.

13. Tkachov, V., \& Savanevych, V. (2014). Method for transfer of data with intermediate storage. In First International Scientific-Practical Conference Problems of Infocommunications Science and Technology, (pp. 105-106), 14-17 October 2014, Kharkiv, Ukraine. DOI: 10.1109/ INFOCOMMST.2014.6992315

14. Lemeshko, O., Yeremenko, O., Yevdokymenko, M., Hailan, A.M., Segeč, P., \& Papán, J. (2019). Design of the fast reroute QoS protection scheme for bandwidth and probability of packet loss in software-defined WAN. In 15th International Conference "The Experience of Designing and Application of CAD Systems (CADSM)”, (pp. 3/72-3/76), 26 February-2 March 2019, Polyana,Ukraine. DOI: 10.1109/CADSM.2019.8779321
15. Lemeshko, O., Yevsieieva, O., \& Yevdokymenko, M. (2018). Tensor flowbased model of quality of experience routing. In IEEE 14th International Conference on Advanced Trends in Radioelecrtronics, Telecommunications and Computer Engineering (TCSET), (pp. 1005-1008), 20-24 February 2018, Lviv, Ukraine. DOI: 10.1109/TCSET.2018.8336364.

16. Lemeshko, O., Yeremenko, O., \& Yevdokymenko, M. (2020). Traffic Engineering Solution of Multipath Fast ReRoute with Local and Bandwidth Protection. In Advances in Computer Science for Engineering and Education II. ICCSEEA 2019. Advances in Intelligent Systems and Computing, (pp.113-125), 29 March 2019. Springer, Cham, vol. 938. DOI:10.1007/978-3-030-16621-2_11

17. Lemeshko, O., Yevdokymenko, M., \& Alsaleem, N. (2018). Development of the Tensor Model of Multipath QoE-Routing in an Infocommunication Network with Providing the Required Quality Rating. Eastern-European Journal of Enterprise Technologies, 5 2(95), 40-46. DOI: 10.15587/1729-4061.2018.141989

18. Lin, S.C., Wang, P., \& Luo, M. (2016). Control Traffic Balancing in Software Defined Networks. Computer Networks, 106, 260-271.

19. Akhtar, Z., \& Falk, T.H. (2017). AudioVisual Multimedia Quality Assessment: A Comprehensive Survey. IEEE Access., 5, 21090-21117. DOI: 10.1109/ ACCESS.2017.2750918

20. Floris, Atzori, L., \& Ginesu, G. (2014). Addressing un-interoperability issues in QoE models: Is a layered modelling effective? In IEEE International Conference on Communications Workshops (ICC), (pp. 563-568), 10-14 June 2014, Sydney, NSW. . DOI: 10.1109/ICCW.2014.6881258

21. Rehman Laghari, K.U., \& Connelly, K. (2012). Toward Total Quality of Experience: A QoE Model in a Communication Ecosystem. IEEE Communications Magazine, 50 (4), 58-65. DOI: 10.1109/ MCOM.2012.6178834 
22. Seppänen, J., Varela, M., \& Sgora, A. (2014). An Autonomous QoE-driven Network Management Framework. Journal of Visual Communication and Image Representation, 25 (3), 565-577.

23. ITU-T G.1070. (2012). Opinion Model for Video-Telephony Applications.

24. Yevdokymenko, M. (2019). Routing tensor model with providing multimedia quality. In International ScientificPractical Conference Problems of Infocommunications. Science and Technology (PIC S\&T), (pp. 1-4), 8-11 October 2019, Kyiv, Ukraine.

25. Kron, G. (1949). Tensor Analysis of Networks. J. Wiley and Sons.

26. Ahmad, A., Floris, A., \& Atzori, L. (2016). QoE-centric Service Delivery: A Collaborative Approach among OTTs and ISPs. Computer Networks, 110, 168-179.
27. Georgopoulos, P., Elkhatib, Y., Broadbent, M., Mu, M., \& Race, N. (2013). Towards network-wide QoE fairness using openflowassisted adaptive video streaming. In SIGCOMM Workshop on Future HumanCentric Multimedia Networking, (pp. 1520), 16 August 2013, Hong Kong, China.

28. Patlins, A., \& Kunicina, N. (2014). The use of remote sensing technology for the passenger traffic flow dynamics study and analysis. In Transport Means - Proceedings of the International Conference, (pp. 63-66), 23-24 October 2014, Kaunas, Lithuania. 